\title{
A New String Model: NEXUS 3
}

\author{
K. Werner ${ }^{1, *}$, F.M. $\operatorname{Liu}^{2,3,1, \dagger}$, S. Ostapchenko ${ }^{4,5}$, T. Pierog ${ }^{6,1}$
}

${ }^{1}$ SUBATECH, Université de Nantes - IN2P3/CNRS - Ecole des Mines, Nantes, France

${ }^{2}$ Institute of Particle Physics, Huazhong Normal University, Wuhan, China

${ }^{3}$ Institut fuer Theoretische Physik, JWG Frankfurt Universitaet, Germany

${ }^{4}$ Institut für Experimentelle Kernphysik, Univ. of Karlsruhe, 76021 Karlsruhe, Germany

${ }^{5}$ Moscow State University, Institute of Nuclear Physics, Moscow, Russia

${ }^{6}$ Forschungszentrum Karlsruhe, Institut für Kernphysik, Karlsruhe, Germany

\begin{abstract}
After discussing conceptual problems with the conventional string model, we present a new approach, based on a theoretically consistent multiple scattering formalism. First results for proton-proton scattering at $158 \mathrm{GeV}$ are discussed
\end{abstract}

\section{Problems with the String Model Approach}

How are string models realized? One may present the particle production from strings via chains of quark lines [1] as shown in fig. 1], It turns out that the two string picture is not enough to explain for example the large multiplicity fluctuations in proton-proton scattering at collider energies: more strings are needed, one adds therefore one or more pairs of quark-antiquark strings, as shown in fig. 2. The variables $x_{i}$ refer to the longitudinal momentum fractions given to the string ends. Energy-momentum conservation implies $\sum x_{i}=1$.

*invited speaker,

9th Winter Workshop on Nuclear Dynamics, Breckenridge, Colorado, February 9 - 14, 2003

${ }^{\dagger}$ Alexander von Humboldt Fellow 

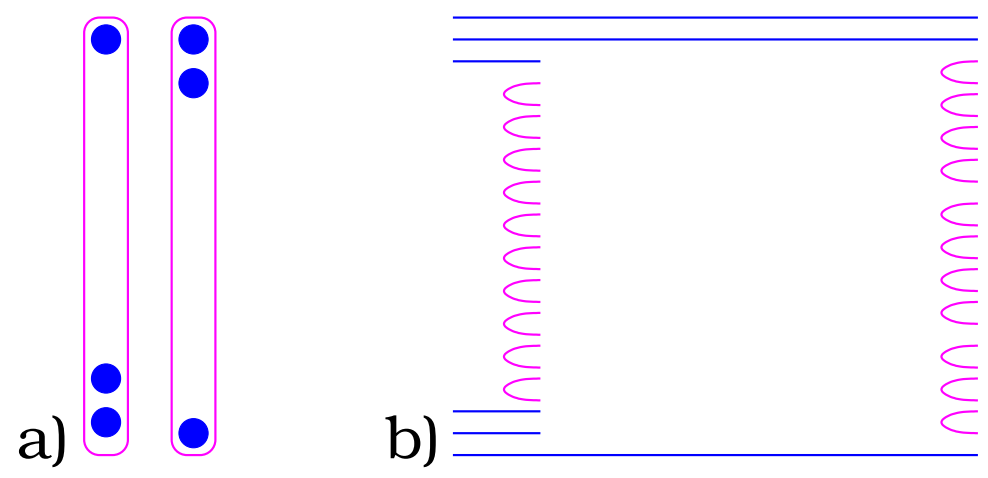

Figure 1: (a) A pair of strings. (b) Two chains of quark lines, equivalent to two chains of hadrons
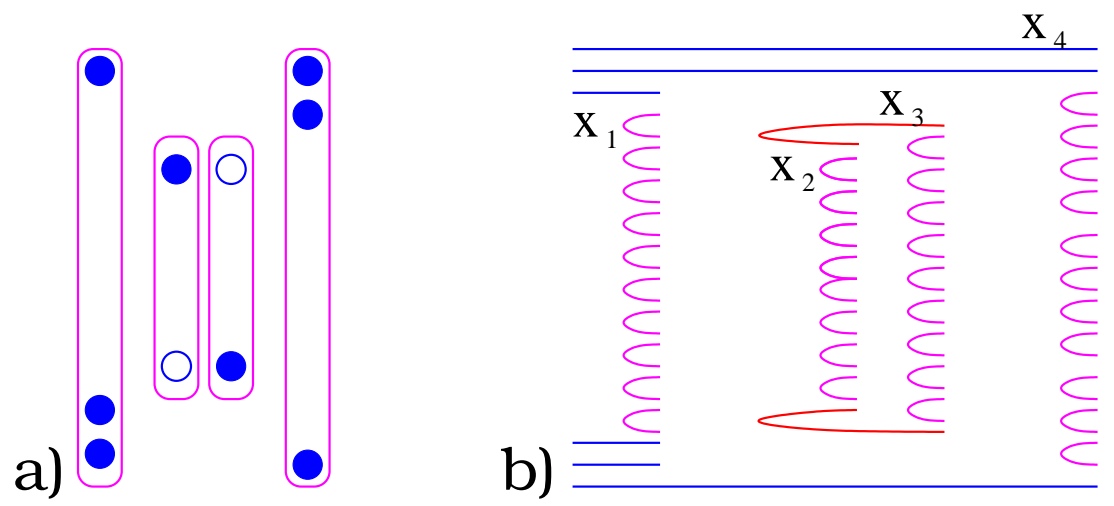

Figure 2: Two pairs of strings (a) and the corresponding chains (b) 
What are the probabilities for different string numbers? Here, GribovRegge theory comes at help, which tells us that the probability for a configuration with $n$ elementary interactions is proportional to $\chi^{n} / n$ !, see fig. 3, where $\chi$ is a function of energy and impact parameter. Here,

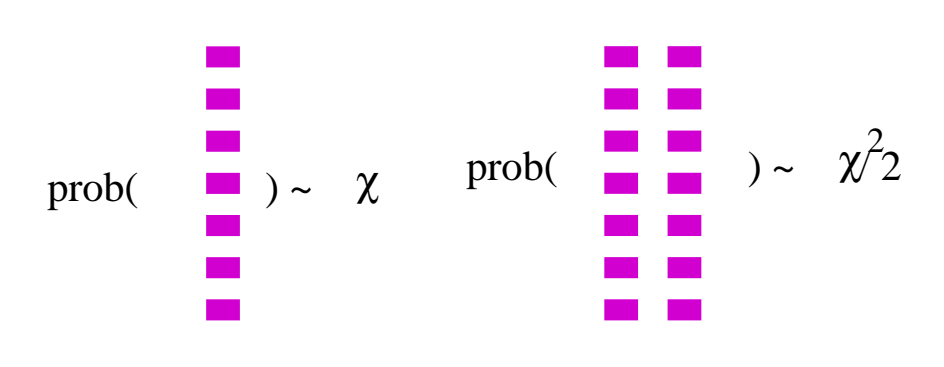

Figure 3: Probabilities for configurations with one and two elementary interactions (Pomerons), represented as dashed lines.

a dashed vertical line represents an elementary interaction (referred to as Pomeron).

Now one identifies the elementary interactions (Pomerons) from GribovRegge theory with the pairs of strings (chains) in the string model, and one uses the above-mentioned probability for $n$ Pomerons to be the probability for configurations with $n$ string pairs. Unfortunately this is not at all consistent, for two reasons:

1. Whereas in the string picture the first and the subsequent pairs are of different nature, in the Gribov-Regge model all the Pomerons are identical.

2. Whereas in the string (chain) model the energy is properly shared among the strings, in the Grivov-Regge approach does not consider energy sharing at all (the $\chi$ is a function of the total energy only)

These problems have to be solved in order to make reliable predictions.

\section{NEXUS}

NEXUS is a self-consistent multiple scattering approach to proton-proton and nucleus-nucleus scattering at high energies. The basic feature is the fact that several elementary interaction, referred to as Pomerons, may happen in parallel. We use the language of Gribov-Regge theory 
to calculate probabilities of collision configurations (characterized by the number of Pomerons involved, and their energy) and the language of strings to treat particle production. We treat both aspects, probability calculations and particle production, in a consistent fashion: In both cases energy sharing is considered in a rigorous way[2], and in both cases all Pomerons are identical. This is one new feature of our approach. Another new aspect is the necessity to introduce remnants: The spectators of each baryon form a remnant. They will play an important role on particle production in the fragmentation region and at low energies $\left(E_{L a b}=40-200 \mathrm{GeV}\right)$. In the following we discuss the details of our approach.

We first consider inelastic proton-proton scattering. We imagine an arbitrary number of elementary interactions to happen in parallel, where an interaction may be elastic or inelastic, see fig. 4. The inelastic amplitude is the sum of all such contributions in with at least one inelastic elementary interaction is involved. To calculate cross sections, we need

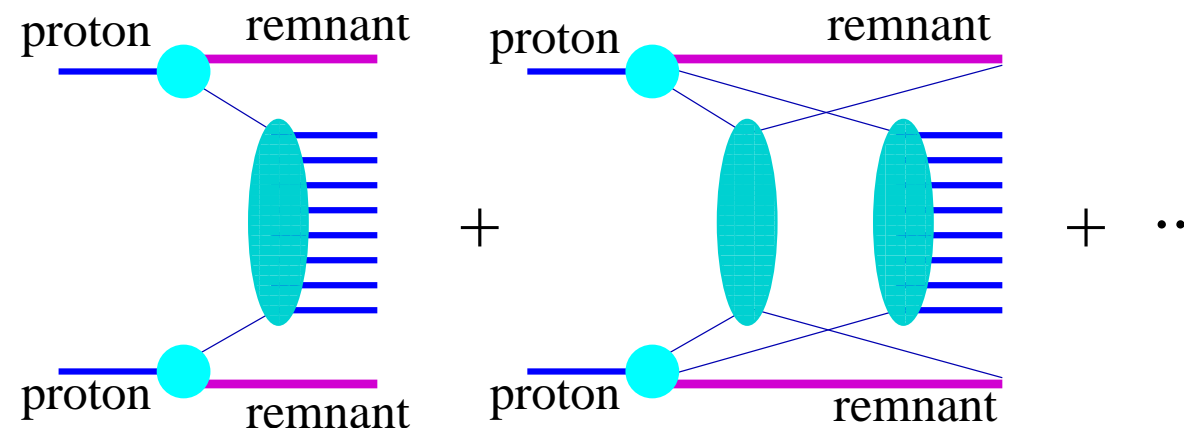

Figure 4: Inelastic scattering in pp. Partons from the projectile or the target proton interact via elementary interactions (the corresponding produced particles being represented by horizontal lines), leaving behind two remnants.

to square the amplitude, which leads to many interference terms, as the one shown in fig. 5|(a), which represents interference between the first and the second diagram of fig. 4, We use the usual convention to plot an amplitude to the left, and the complex conjugate of an amplitude to the right of some imaginary "cut line" (dashed vertical line). The left part of the diagram is a cut elementary diagram, conveniently plotted as a dashed line, see fig. 5(b). The amplitude squared is now the sum over many such terms represented by solid and dashed lines.

Summing appropriate classes of interference terms, we obtain prob- 

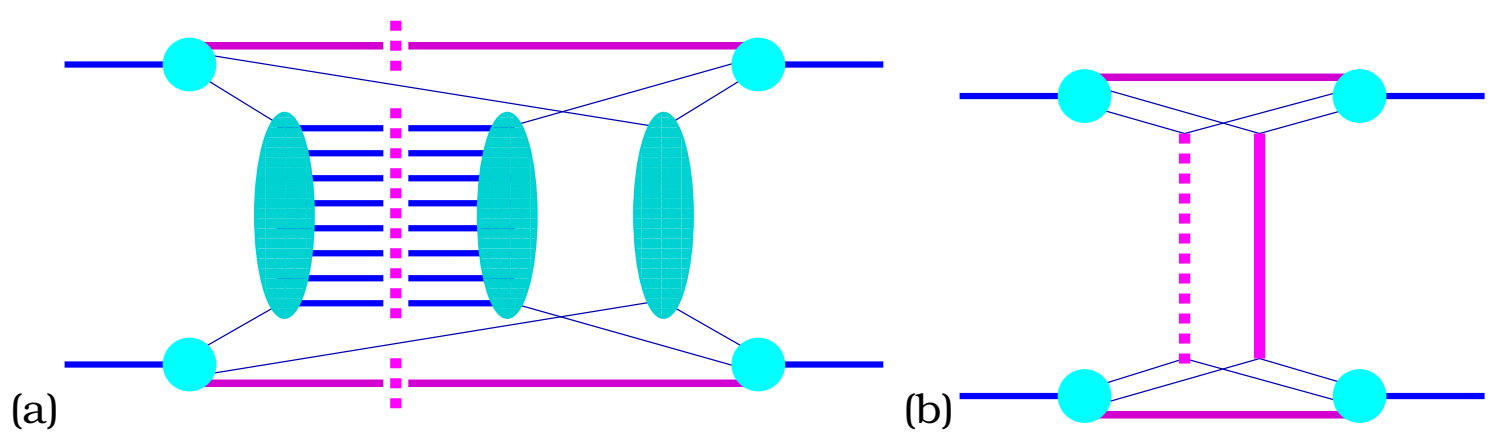

Figure 5: Inelastic scattering in pp. a) An interference term , b) in simplified notation.

abilities of having $m$ inelastic interactions with light cone momenta $x_{1}^{+} . . x_{2 m}^{+}, x_{1}^{-} \ldots x_{2 m}^{-}$at a given impact parameter. Integrating over impact parameter provides the corresponding cross section. By this we obtain a probability distribution for the number of elementary interactions (number of Pomerons) and the momenta of these Pomerons.

How to form strings from Pomerons? No matter whether singlePomeron or multiple-Pomeron exchange happens in a proton-proton scattering, all Pomerons are treated identically. Each Pomeron is identified with two strings.

The string ends are quarks and antiquarks from the sea. This differs from traditional string models, where all the string ends are valence quarks. Due to the large number of Pomerons this is impossible in our approach. The valence quarks stay in remnants. Being formed from see quarks, string ends from cut Pomerons have complete flavor symmetry and produce particles and antiparticles in equal amounts.

Remnants are new objects, compared to other string models, see fig.6. A remnant contains three valence quarks and the corresponding antiparticles of the partons representing the string ends. We parametrize the mass distribution of the remnant mass as $P\left(m^{2}\right) \propto\left(m^{2}\right)^{-\alpha}$, where $m$ is taken within the interval $\left[m_{\min }^{2}, x^{+} s\right]$, with $s$ being the squared total energy in the center of mass system, $m_{\min }$ being the minimum mass of hadrons to be made from the remnant's quarks and antiquarks, and $x^{+}$ being the light-cone momentum fraction of the remnant. Through fitting the data at $158 \mathrm{GeV}$, we determine the parameter $\alpha=2.25$. Remnants decay into hadrons according to n-body phase space 3].

The most simple and most frequent collision configuration has two remnants and only one cut Pomeron, represented by two $q-\bar{q}$ strings 


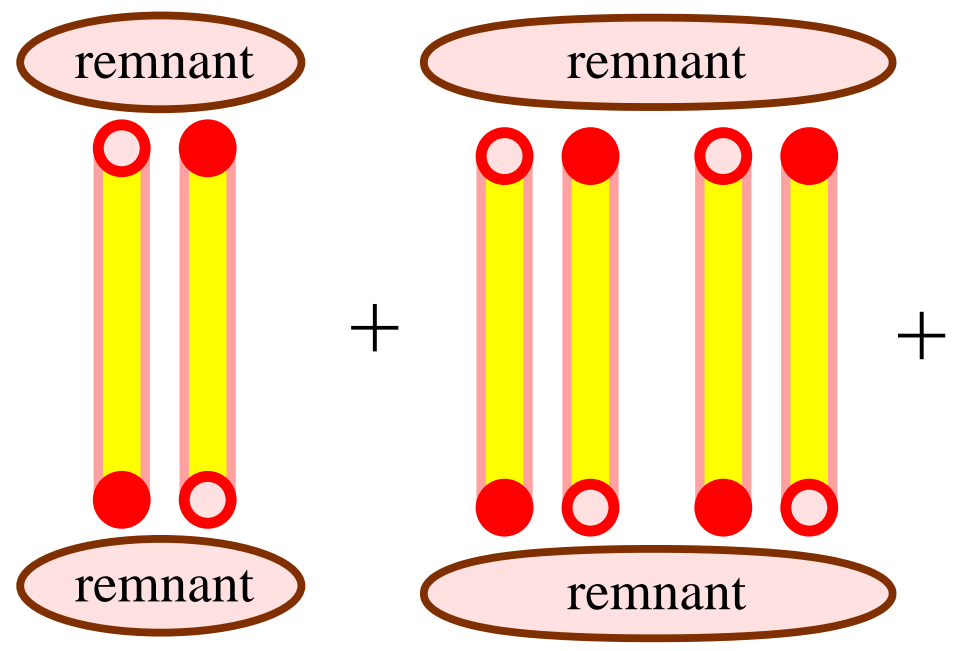

Figure 6: Remnants in single (two strings) and double scattering (four strings): in any case, two remnants contribute.

as in Fig:7(a). Besides the three valence quarks, each remnant has in addition a quark and an antiquark to compensate the flavor.

We assume that an antiquark $\bar{q}$ from a string end may be replaced by a diquark qq, with a small probability $P_{q q}$. In this way, we get quarkdiquark $(\mathrm{q}-\mathrm{qq})$ strings from cut Pomerons. The qqq Pomeron end (the sum of the two corresponding string ends) has to be compensated by the three corresponding antiquarks in the remnant, as in Fig.7(b). The (3q3 $\bar{q})$ remnant decays according to phase space, so mainly into three mesons (3M), and only with a very small probability into a baryon and an anti-baryon $(\mathrm{B}+\overline{\mathrm{B}})$. For symmetry reasons, the $\mathrm{q}$ string end is replaced by an antidiquark $\overline{\mathrm{qq}}$ with the same probability $P_{\mathrm{qq}}$. This yields a $\overline{\mathrm{q}}-\overline{\mathrm{qq}}$ string and a (6q) remnant, as shown in Fig.7(c). The (6q) remnant decays into two baryons. Since q-qq strings and $\bar{q}-\overline{\text { qq }}$ strings have the same probability to appear from cut Pomerons, baryons and antibaryons are produced in the string fragmentation with the same probability. However, from remnant decay, baryon production is favored due to the initial valence quarks.

With decreasing energy the relative importance for the particle production from the strings as compared to the remnants decreases, because the energy of the strings is lowered as well. If the mass of a string is lower than the cutoff, it will be discarded. However, the fact that an interaction has taken place is taken into account by the excitation of the remnant, which follows still the above mentioned distribution. 

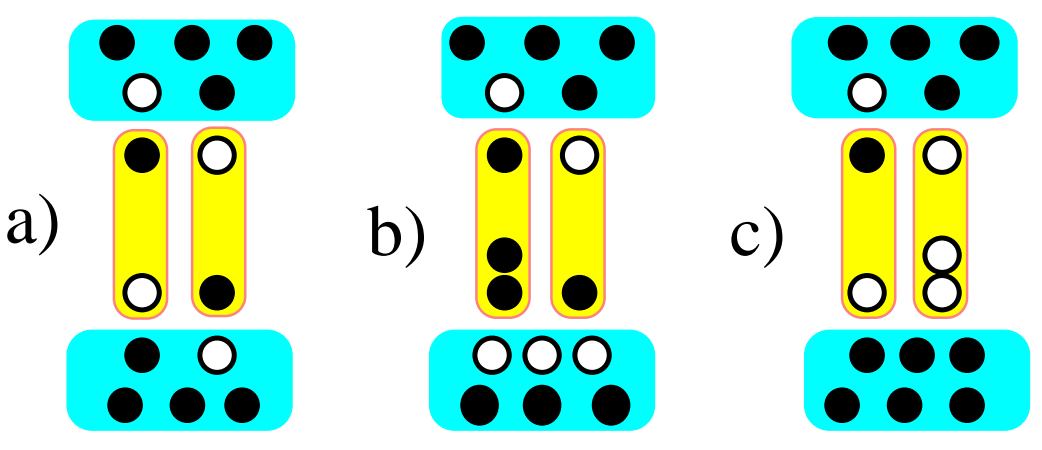

Figure 7: (a) The most simple and most frequent collision configuration has two remnants and only one cut Pomeron represented by two $q-\bar{q}$ strings. (b) One of the $\bar{q}$ string-ends can be replaced by a qq string-end. (c) With the same probability, one of the q string-ends can be replaced by a $\overline{q q}$ string-end.

\section{Results}

Fig. 8 shows the rapidity spectra for pp at $158 \mathrm{GeV}$. We included the $\Lambda, \bar{\Lambda}, \Xi$, $\Xi$ spectra, which have been published earlier |4|. Where data from the NA49 collaboration are available, we have included them in the plot[6]. The yield gives the calculated average multiplicity of the particle species in $4 \pi$. We see that the experimental data are reasonable described. The non strange baryons as well as those which contain one strange quark show a double hump structure, the others are peaked at mid rapidity. This is a consequence of the three source structure (two remnants and Pomerons) in our approach. The leading baryon has still the quantum number of the incoming baryon but is moderately excited. Therefore it may disintegrate into baryons whose quantum numbers differ not too much.

We observe in particular more $\Omega$ than $\bar{\Omega}[5]$, as seen in experiment [7]. This is a consequence of the modification of NEXUS 3, explained in 44, as compared to the original NEXUS 2 version [2] (and many other string models), which yields more $\bar{\Omega}$ than $\Omega$ due to the string topology .

\section{References}

[1] J. Ranft, Z. Phys. C 43 (1989) 439; A. Capella et al., Phys. Rep. 236 (1994) 225 
$\mathrm{p}+\mathrm{p}$ at $\mathrm{E}_{\mathrm{lab}}=158 \mathrm{GeV}$
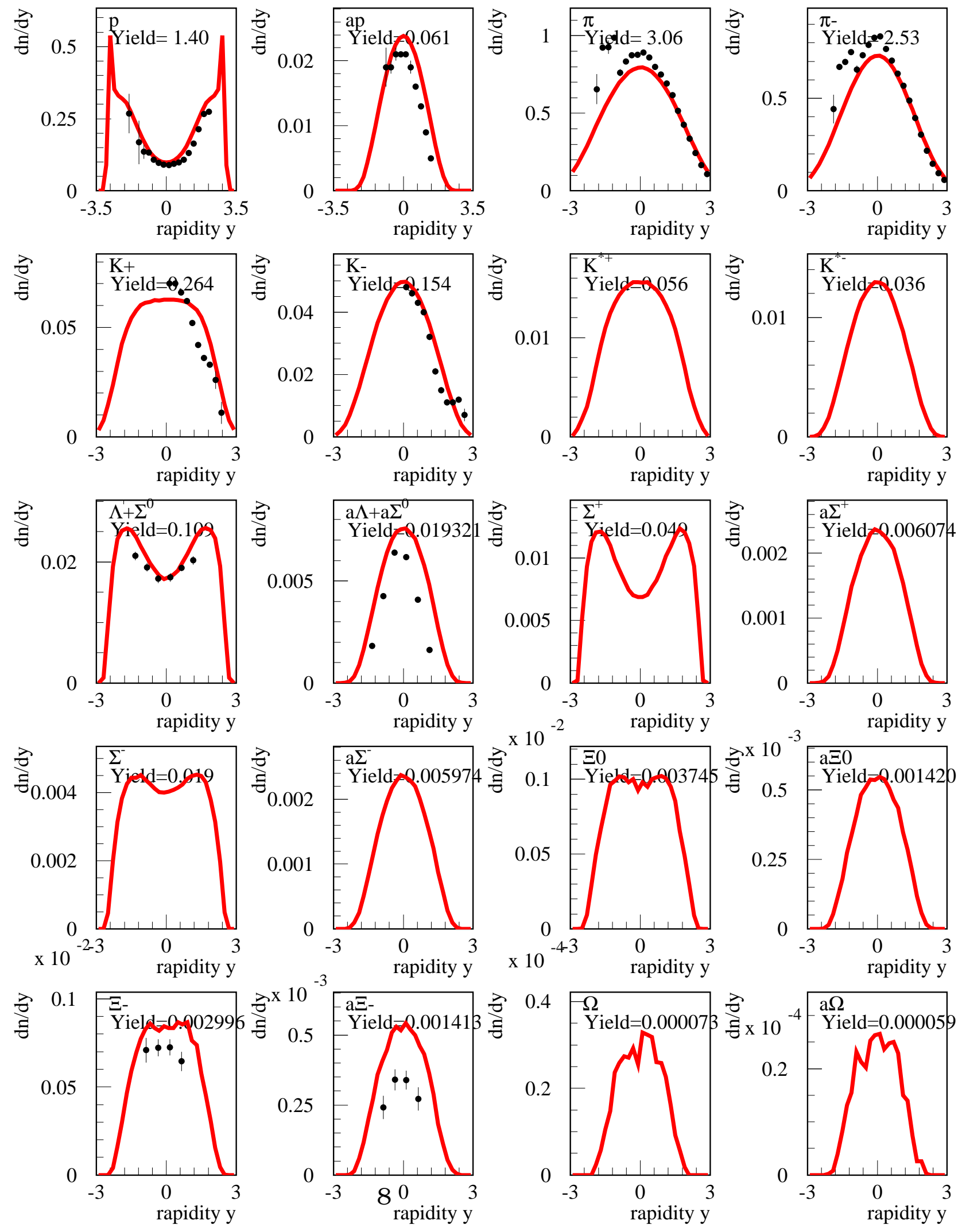

Figure 8: Proton-proton at $158 \mathrm{GeV}$ 
[2] H.J. Drescher, M. Hladik, S. Ostapchenko, T. Pierog, K. Werner, Phys.Rep. 350 (2001) 93

[3] K. Werner and J. Aichelin, Phys. Rev. C 52 (1995) 9503021.

[4] F.M. Liu, J.Aichelin, M.Bleicher, H.J. Drescher, S. Ostapchenko, T. Pierog, K. Werner, Phys. Rev. D67 (2003) 034011

[5] M. Bleicher, F. M. Liu, A. Keränen, J. Aichelin, S.A. Bass, F. Becattini, K. Redlich, K. Werner, Phys. Rev. Lett. 88 (2002) 202501

[6] NA49 collaboration, homepage

[7] Mischke for the NA49 collaboration, Nucl. Phys. 698 (2002) 491c 